\begin{tabular}{|c|c|c|}
\hline$\overbrace{\text { INESEG }}$ & $\begin{array}{c}\text { International Journal of Health Services } \\
\text { Research and Policy }\end{array}$ & \\
\hline $\begin{array}{c}\text { INTERNATIONAL } \\
\text { ENGINEERING }\end{array}$ & www.dergipark.org.tr/ijhsrp & \\
\hline $\begin{array}{c}\text { SCIENCE AND } \\
\text { EDUCATION GROUP }\end{array}$ & e-ISSN: 2602-3482 & IJHSRP \\
\hline
\end{tabular}

Research Article

\title{
A QUALITATIVE STUDY ON NEEDS AND BARRIERS TO MENTAL HEALTHCARE SERVICE ACCESS BY REFUGEE WOMEN IN WINNIPEG, MANITOBA, CANADA
}

\author{
Sanjida Newaz ${ }^{* 1,2}$ (i) Natalie Riediger ${ }^{1,3}$ (D) \\ ${ }^{1}$ Department of Community Health Sciences, Max Rady College of Medicine, University of Manitoba, \\ Winnipeg, MB, Canada \\ ${ }^{2}$ Saskatchewan Health Authority, Regina, SK, Canada \\ ${ }^{3}$ Department of Food and Human Nutritional Sciences, Faculty of Agricultural and Food Sciences, University of \\ Manitoba, Winnipeg, MB, Canada \\ *Corresponding author; newazs@ myumanitoba.ca
}

\begin{abstract}
The world refugee crisis is currently at a record level. Refugees have an increased risk of developing a mental illness like post-traumatic stress disorder (PTSD), depression, and somatic symptoms because of their exposure to violence. Despite increased healthcare needs, refugees face considerable barriers in accessing services. Women refugees may have unique mental healthcare needs due to their vulnerability to gender-based violence and abuse during flight from war. This study explores the mental healthcare needs and barriers in accessing services among Syrian refugee women in Winnipeg. Semi-structured interviews were conducted with nine refugee women and six service providers/decision-makers. The data were analyzed using a qualitative thematic approach aided by NVivo 12 qualitative software. The most cited barriers in accessing mental healthcare services were language, weather, unemployment, stigma, system navigation, different understandings of mental health and illness, and lack of culturally competent care. Results from this study can inform decision-makers of issues requiring policy responses to improve mental healthcare for refugee women in Manitoba.
\end{abstract}

Keywords: Refugee women, mental healthcare, access barriers, qualitative research

Received: September 23, $2020 \quad$ Accepted: November 25, 2020

\section{Introduction}

The civil war in Syria has created a humanitarian crisis, probably the worst in our lifetime. Over 6.7 million Syrians have fled their country to seek safety, mostly in the neighbouring countries Lebanon, Turkey, Jordan, Iraq, as the United Nations High Commissioner for Refugees (UNHCR) reports [1]. The Government of Canada responded to the humanitarian crisis in Syria by welcoming 25,000 refugees within 100 days between November 4, 2015, and February 29, 2016, under the project named 'Operation Syrian Refugees' [2]. Canada worked with UNHCR to identify vulnerable refugees in Jordan and Lebanon, who were also a low-security risk, such as women at-risk and complete families. The commitment to resettling Syrian refugees continued in 2017, and 40,081 refugees were resettled in Canada as of January 29, 2017 [3]. 
The UNHCR reported the most prevalent mental health problems among Syrian refugees as depression, prolonged grief disorder, post-traumatic stress disorder (PTSD), and various forms of anxiety disorders [4]. Lack of access to healthcare prior to migration, prolonged and poor living conditions in camps, a journey during migration, and overcrowded reception centers contribute to the poor health conditions of refugees [5]. As refugees arrive in safety and protection in Canada, the struggles do not end there since new challenges begin during their settlement and integration process [6], further compromising mental wellbeing. Social causes of mental illness often referred to as social determinants of health, such as gender roles and identities, age, unemployment, and income insecurity, substandard living conditions, intergenerational struggles, isolation, racism, and discrimination, can make the integration process very difficult and exacerbate the mental health condition of refugees [7][9]. Not speaking English, cultural differences, lower socio-economic status, reluctance to disclose mental illness, religious beliefs, and many other barriers may further limit access to healthcare [10]. Women refugees may have unique mental healthcare needs due to their vulnerability to gender-based violence and abuse during flight from war [11]-[13]. The effects of war, family separation, displacement, and long travel may pose additional stresses on women, particularly when coinciding with pregnancy, childbirth, and caring for children [7], [12], [14]. Moreover, women refugees may be disproportionately challenged by social exclusion and isolation as compared to men as they are less likely to speak the language of the new country and be employed outside the home [9], [14]. Refugee women may not actively seek help, therefore potentially increasing their risk of developing long-term mental health issues or illness.

The Canadian province of Manitoba resettled a record 3,730 refugees in 2016 [15]. Winnipeg is the largest city in the province and the vast majority of its population live there. Considering the recent influx of refugees, it is important to understand how Manitoba is addressing the mental health needs of refugee women. In recent years, the healthcare services in Manitoba are experiencing major funding decreases and policy reforms, which may bring changes to how the mental healthcare services are offered in the province [16]-[18]. While the mental healthcare needs of refugees, especially refugee women, are significant, there is a lack of detailed research into access to mental healthcare services for refugee women in Manitoba and Canada. Most studies focus on immigrants, which includes refugees. However, there is a clear distinction between the two groups - immigrants are people who choose to come to live permanently in a foreign country whereas refugees are forced to flee their country of origin to seek safety in another country. Therefore, mental healthcare needs can not be generalized for all immigrants and refugees, especially when considering the past lived experience. No study is available on the mental healthcare needs of refugee women in Winnipeg or Manitoba. Although studies conducted in Winnipeg found evidence of mental health issues in many refugees and the challenges they face while accessing mental healthcare services [7], [19], [20], service providers and decision-makers were not included to examine their perspectives on refugee mental healthcare issues. This study, by exploring refugee women's mental healthcare needs and barriers to accessing services in Winnipeg, will bring the voices of refugee women to the attention of policymakers, as to the kinds of services they need, the services available to them, and the ways they now access services. Results from this study may be used to identify existing gaps in services and inform the ongoing development of services and support for refugees 


\section{Background}

\subsection{Refugee Settlement Statistics in Manitoba}

Manitoba has welcomed an average of 1,250 refugees per year between 2006 to 2017 [15], [21]. The province accepted a record 3,730 refugees in 2016 and a 5-year average of over 1,900 refugees. In 2012 , the number of refugees' arrival to the province decreased mainly due to the federal cap imposed on the Manitoba Provincial Nominee Program (MPNP) [21]. The increased processing times at Immigration Refugees and Citizenship Canada (IRCC) was also a contributing factor. However, after 2012 the number of refugees in the province continued increasing. In fact, the 2013 - 2017 data from IRCC shows that Manitoba is accepting the highest number of refugees per capita than any Canadian province or territory (Figure 1). According to the last statistical report on immigration facts published by the Ministry of Labour and Immigration [22], Manitoba settled nearly 6\% of Canada's governmentassisted refugees and $22 \%$ of privately sponsored refugees in 2014. In 2016, the majority of refugees to Manitoba came from Syria. Most refugees to Manitoba settled in Winnipeg, therefore the city is facing major challenges in meeting the complex physical and emotional issues and needs of refugees [21].

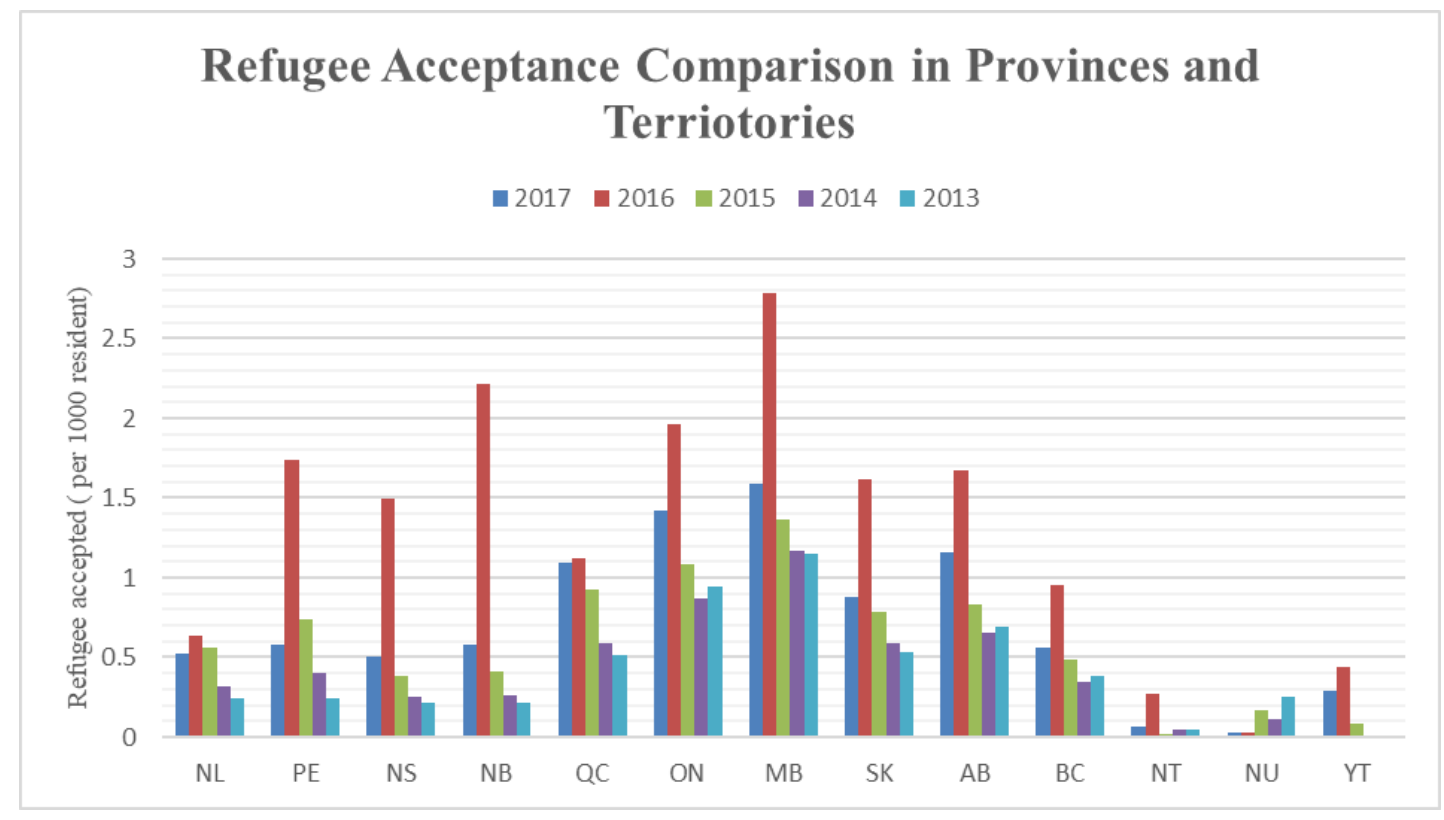

Figure 1. Refugee Acceptance Comparisons among Canadian Provinces and Territories [15] $* \mathrm{NL}=$ Newfoundland and Labrador, $\mathrm{PE}=$ Prince Edwards Island, NS $=$ Nova Scotia, NB $=$ New Brunswick, $\mathrm{QC}=$ Quebec, $\mathrm{ON}=$ Ontario, $\mathrm{MB}=$ Manitoba, $\mathrm{SK}=$ Saskatchewan, $\mathrm{AB}=$ Alberta, $\mathrm{BC}=$ British Columbia, $\mathrm{NT}=$ Northwest Territories, $\mathrm{NU}=$ Nunavut, $\mathrm{YT}=$ Yukon

\subsection{Available Mental Healthcare Resources in Manitoba}

In Manitoba, mental healthcare services are provided through the local Regional Health Authorities' (RHA) mental health program. The Bridge Care Primary Clinic in Winnipeg provides a single point of access for the initial health assessment and primary care services for newly arrived government-sponsored refugees [23], [24]. The clinic also makes referrals for mental health services of which there are several mental healthcare service providers in Winnipeg, including mental healthcare services offered specifically to refugees. Mental healthcare services are also available at Winnipeg's major hospitals and urgent care facilities. Some hospitals have psychiatric nurses on duty in emergency. 
While there are many programs and services available in Winnipeg, the effectiveness of these initiatives remains unclear. The supporting services may have been stretched to their limits and facing staffing shortages due to the recent record number of refugee influx in the province. Literature suggests that how refugees perceive the available mental healthcare services and how healthcare providers offer such services should be examined to understand refugee mental health needs and services [19], [25], [26]. In this study important information regarding the knowledge and accessibility of mental health services among refugee women in Winnipeg was obtained by interviewing refugee women, service providers, and decision-makers in Winnipeg.

\section{Materials and Methods}

\subsection{Research Design}

A qualitative research design was employed to explore the mental healthcare service accessibility issues of Syrian refugee women living in Winnipeg. Semi-structured interviews were conducted with refugee women and service providers/decision-makers living/working in Winnipeg. Interviews with the refugee women provided their perspective on mental wellbeing, healthcare needs, and barriers to accessing mental health services. Interviews with the service providers/decision-makers provided useful information on the need for culturally competent healthcare, collaboration among providers, funding, and provincial healthcare strategies. Organizations such as, settlement agencies and clinics that welcome and serve refugees in Winnipeg, were contacted to collaborate in recruitment and provide input in preparing the interview guides. The individual interviews were planned such that both the providers and refugee women's interviews were conducted concurrently. The interview guides were piloted first and then were updated, as necessary. Initial analyses and interviews also informed subsequent interviews so that it was possible to build on and further explore emerging themes.

Ethics approvals were obtained from (1) Health Research Ethics Board (HREB), University of Manitoba and (2) Research Access and Approval Committee, Winnipeg Regional Health Authority (WRHA). (Approval date: November 15,2018; Approval Number: H2018:406)

\subsection{Sample and Recruitment}

Nine Syrian refugee women were recruited for interviews. Eligibility criteria included refugee women who have settled in Winnipeg within the last 1 to 5 years and were between 18 - 45 years old. It was believed that women within this age range are more likely to seek employment, participate in EAL classes or other educational programs including colleges and universities, undertake more family responsibilities, and therefore would face challenges in accessing mental healthcare services. Moreover, women of this age group may have more interest in participating in interviews. Consideration was given to recruiting refugee women who spent a considerable amount of time (1 to 5 years) in Winnipeg after resettlement, therefore have accessed or had experience with mental healthcare services. The refugee women were recruited for interviews using a combination of convenience and snowball sampling. First, a list of refugee settlement organizations and specialized refugee primary care clinics in Winnipeg was prepared through an online search. Second, representatives of these organizations were contacted via sending email invitations followed by an in-person visit. Refugee support service organizations were requested to coordinate and facilitate the recruitment of their Syrian clients (convenience sampling). 
Third, at the end of each interview, the refugee women were asked to recommend other potential participants for this study (snowball sampling).

Table 1 provides an overview of the participant characteristics. Among nine refugee women, six were married, two were widowed and one was single. All but one refugee woman disclosed their age, which ranged from 19 - 41 years, with family sizes ranging from 1-9. The education level among the refugee women participants varied. One refugee woman had a bachelor's degree from Syria and working part-time or in a temporary position in Winnipeg. One refugee woman was attending a post-secondary institution. The remaining seven participants had education levels in between elementary to Grade 9 and the majority mentioned attending English as an Additional Language (EAL) programs in Winnipeg. One refugee woman was employed, and another engaged in casual work. The majority of the participants had to rely on social assistance for living. Two participants were privately sponsored refugees (PSRs) and the remaining seven were government-sponsored refugees (GSRs). By reaching a sample size of nine, a wide range of participant characteristics was covered - such as PSRs and GSRs, employed and unemployed, single/widowed refugee women, and refugee women having spouses, large and small families, varying education background, etc.

Representatives from organizations who were contacted to help with recruiting refugee women were also requested for interviews as service providers either by themselves or by referring to their coworkers. In addition, personal communication and references were used to recruit potential interview participants. Manitoba Health, Seniors, and Active Living (MHSAL) were contacted to take part as a decision-maker. Five service providers and one decision-maker consented to participate.

Table 1. Interview Participants

\begin{tabular}{|c|c|c|}
\hline Category & $\begin{array}{l}\text { Participant } \\
\text { Number }\end{array}$ & Participant Details \\
\hline $\begin{array}{l}\text { Syrian Refugee Women } \\
\text { (RW) }\end{array}$ & 9 & $\begin{array}{l}\text { - } 2 \text { Privately sponsored and } 7 \text { Government sponsored } \\
\text { - } 2 \text { were able to communicate in English and } 7 \text { participated } \\
\text { through interpreters } \\
\text { - The duration of the settlement was } 16 \text { to } 36 \text { months }\end{array}$ \\
\hline $\begin{array}{l}\text { Service Providers and } \\
\text { Decision Makers (SP) }\end{array}$ & 6 & $\begin{array}{l}\text { Professionals who directly or indirectly serve refugees such as } \\
\text { Psychiatrist, Family Physician, Settlement Worker, Decision } \\
\text { Maker }\end{array}$ \\
\hline
\end{tabular}

\subsection{Data Collection}

The refugee women were offered one-on-one interviews at a time and a place of their convenience with the first author. Two out of nine Syrian refugee women were able to communicate in English. Two professional interpreters were required for the remaining seven interviews. Both interpreters were women and working at refugee serving organizations, but not from Syria. Service providers and decision-makers were offered options to participate in person, or via teleconference, based on the location and convenience. Informed consent was obtained from all participants before the interviews. Most of the interviews with refugee women were conducted at the refugee serving organizations, which may have further enhanced their comfort since they are familiar with space and environment. Initial analyses and interviews also informed subsequent interviews so that it was possible to build on and further explore emerging themes. The interviews were conducted between February to May 2019 and 
lasted for approximately 40 - 90 minutes. All interviews were audio-recorded with permission and transcribed verbatim.

\subsection{Analysis}

The data were analyzed using qualitative analysis and coded for themes based on recurring issues aided by NVivo 12 qualitative software. Qualitative thematic analysis - which is a method for identifying, analyzing, organizing, describing, and reporting themes found within a data set [27], [28] was best suited for this study. Braun and Clarke (2006) presented thematic analysis as a linear, sixphased method which includes - (a) Familiarizing with Data, (b) Generating Initial Codes (c) Searching for Themes, (d) Reviewing Themes, (e) Defining and Naming Themes and (f) Producing the Report [27]. Additionally, the analysis met Lincoln and Guba's (1985) five criteria for trustworthinesscredibility, transferability, dependability, confirmability, and audit trails [29].

The interviews for both refugee women and service providers were analyzed simultaneously. In summary, the analysis process included reading the transcripts multiple times to familiarize with the data. Then a list of primary codes was developed inductively. The codes were further refined, expanded, and combined to form overarching themes. While reviewing, defining, and naming themes, sub-themes were assigned within themes where similarities among codes were found. In addition to the analysis software NVivo 12, suggestions from Braun and Clarke (2006) such as visual representations (mindmaps), and other techniques (tables, writing down the codes in a separate piece of paper and sorting the piles of developed themes, etc.) were implemented during the analysis [27].

\section{Results}

\subsection{Mental Healthcare Needs of Refugee Women and Sources of Stress}

The mental health issues that the Syrian refugee women talked about during the interviews were lack of overall mental wellbeing, anxiety, frustration, stress, and persistent sadness. Additional mental health concerns that the service providers identified among refugee women were trauma, mood issues, sleep problems, PTSD, and grief. The most common sources of stress identified among refugee participants were not speaking the English language, unemployment, health issues of family members, difficulties in terms of relationships with children, fear of negative repercussions (such as losing custody of children to Child and Family Services), struggles of settling in a new country, weather, bullying, the feeling of being unwelcomed, and family members left behind.

\subsection{Barriers in Accessing Mental Healthcare Services}

The barriers in accessing mental healthcare services were grouped into three themes as illustrated in Figure 2: material; structural and organizational; and social and cultural. Each theme is described in detail below. Importantly, there were also inter-relationships between some themes, which are also highlighted below. Lastly, what were described as barriers to accessing mental health services were, in many cases, simultaneously sources of stress, which potentially contributed to poorer mental health.

\subsubsection{Material Aspects}

(a) Employment and Income Level 
Most of the refugee women interviewed did not work in the labour force and were relying on income assistance. Refugee women who are widowed and/or have children to take care of, face more challenges as their family roles begin to change and the traditional support system they had at home becomes non-existent. With increased family responsibilities, some work multiple jobs and do not have any time for self-care.

"The life is very hard over there, I mean the financial, I had to take care of everything. And, because I'm by myself, the responsibility is too big" ( $R W$ 5)

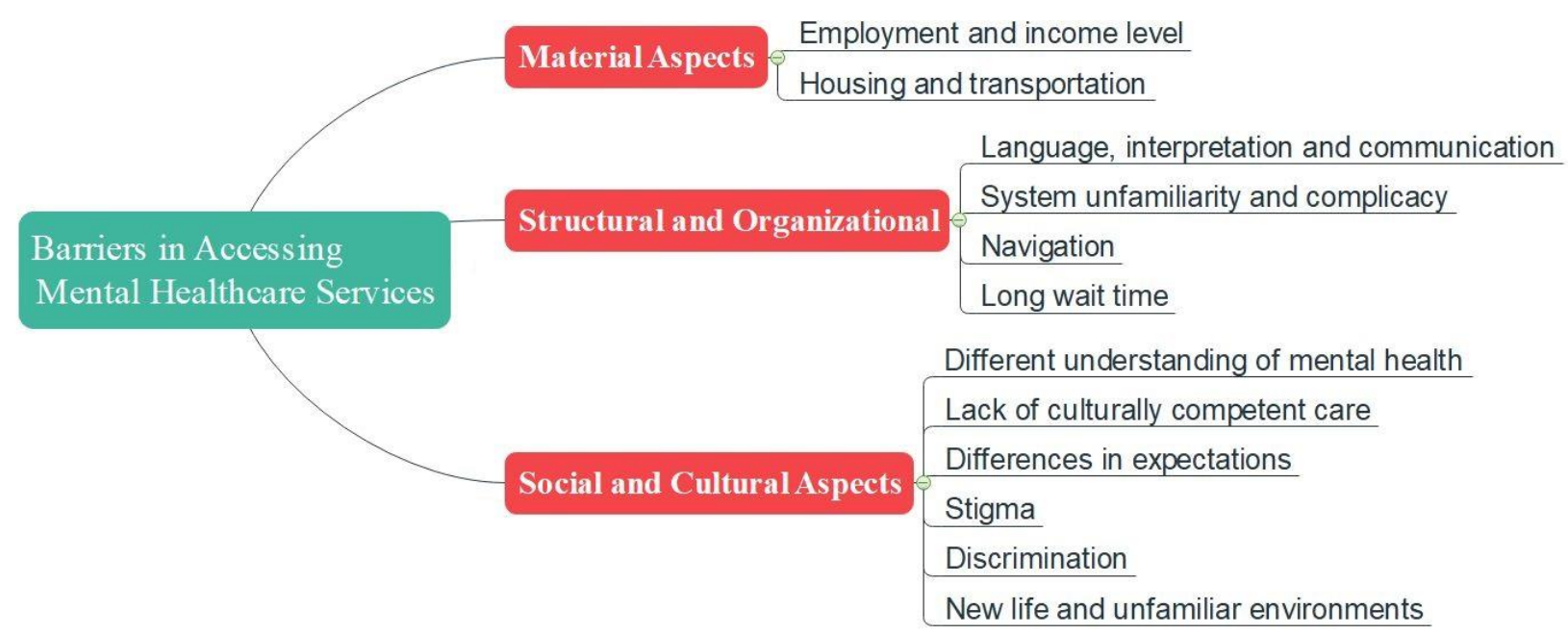

Figure 2. Mind Map of Barriers Faced by Refugee Women in Accessing Mental Healthcare

(b) Housing and Transportation

Immigrant \& Refugee Community Organization of Manitoba (IRCOM) helps the refugee women to find affordable housing, usually centrally located in Winnipeg. Being located close to downtown, the participants described having better access to all services while living in these places. However, they need to move out after a certain period (usually 2-3 years). Several refugee women mentioned moving to the south side of the city and facing barriers in accessing services like transportation, distance to the facilities, etc. Both the refugee women and service providers also discussed the challenging weather conditions. The cold weather during winter makes it even more difficult to use public transit if they want to visit a doctor or therapy/counselling service provider.

"Among other barriers the cold, the winter is really bad for mobility. Especially we have a lot of people who have challenges, like physical challenges, wheelchair dependent. Some have a hard time to connect to handi-transit, which always takes time. So, mobility is a big thing” (SP 5)

Since the Health Sciences Center (HSC) (the largest hospital facility in the city of Winnipeg) and the majority of the service-providing organizations are located near the downtown area, participants talked about the lack of service facilities in other parts of the city, such as in the south end. Carrying disabled children to doctor's offices is another major challenge that a participant pointed out.

"For me, it was a challenge to take my children by myself, especially my two children, their physical disability - they can not walk" (RW2) 
For one participant, housing costs kept her so occupied that she was unable to think about her own mental health or to see a doctor. Financial inability limits the refugee women's access to better housing, in terms of both renting and purchasing, and hence was also a source of mental stress.

"The rent is very expensive and compares to our country, everything over there was very cheap, and here it is very expensive. We cannot afford it." (RW 6$)$

\subsubsection{Structural and Organizational}

(a) Language, Interpretation, and Communication

The language barrier is one of the major challenges in accessing any healthcare services that was repeatedly mentioned - in the literature, by the service providers, and the refugee women themselves.

"The first issue is language. Somebody is talking to you and you cannot respond to them. You don't understand anything. It is not easy." (RW 6)

The service providers discussed this issue as well. It is not easy for refugee women to learn a new language so that they can communicate with the service providers or in accessing services beyond healthcare. Moreover, as discussed in the literature, gender further complicates one's access to learn English, which the service providers found to be true.

"Language issue is a problem, especially in a lot of cultures women are not educated, they don't speak English, they have a hard time learning English because they are illiterate. Especially if they are single women with children and if there is no male figure around, it's harder for them to learn the language, navigation, as they try to make the appointments or going to the specialist appointment can be very difficult and challenging." (SP 5)

Due to the lack of English language fluency many refugee women need the assistance of an interpreter to communicate with mental health professionals. Needing the help of an interpreter means that someone else must attend appointments with the refugee women to translate, and the availability or wait times for that individual also adds to the wait time to see a psychiatrist or psychologist.

"The bigger issue we have here is language, it's a barrier. We always need somebody to be with us to translate." (RW 7)

Since professional interpretation services are not always available, options like using family members including children, friends, personnel from the same community or culture, telephone interpretation services, etc. have been explored by service-providing organizations. They all have disadvantages, as the service providers have pointed out.

"Sometimes we use telephone interpreters, which then could be from anywhere in North America.

It's much more anonymous but it may not be as effective because you don't have the person in the room to read the facial expressions and know what's going on". (SP 2)

The service providers have noticed that many of their clients didn't like using the telephone and felt that therapy was enhanced by having an in-person interpreter in the room. Many refugee women were not comfortable sharing their mental health issues or past experiences in front of an interpreter who is from their same community. This also applies to using friends or family members. Therefore, on many occasions, refugee women have searched for service providers who speak the same language. 
(b) System Unfamiliarity and Complicacy

The lack of knowledge about the Canadian healthcare system, in general, is a challenge for refugees. To see a specialist, for example, a Psychiatrist involves many steps like seeing a Family Physician or a Primary Care Physician first, then getting referred, making appointments, etc. The service providers have recognized that the system is complex for the refugee women and very different than what they have been used to.

"But the system is too complex. This one is for everyone, its not only for refugees." (SP 1)

"The system is different here. I think a lot of refugees are kind of lost about how the system works here. We try to tell them and explain to them in their first visit what is the emergency for, when should they go to the family doctor. But I think it's very challenging for them to get used to that, to learn the system. So, we need more communication, collaboration. Healthcare system is for sure a barrier." (SP 5)

(c) Navigation

System navigation is particularly challenging for refugee women, especially those who do not have a male figure around them. The refugee women recognized that there are services available for them, but they do not know how to get there, especially using public transit. Many depend on their husband to take them for appointments as they cannot navigate by themselves.

"I depend on him; because 2 weeks ago I tried to go into my early morning job, I wanted to go by myself. I just crossed the street, I slipped, I lost my bus pass, and the bus went (left). I went back home to wake up my daughter, I told her to give me a bus ticket, then I again went back. At that time, I realized how I'll deal/ work without him (my husband)." (RW 3)

"I can not find my way by using buses or knowing the addresses. The language is a big problem. Even there may be services out there, but I don't know how to reach to those services." (RW I)

(d) Long Wait Time

Long wait time is a major problem for the overall healthcare system in Canada. Both the refugee women and services providers discussed this particular issue. The long wait time is a significant barrier as it demotivates the refugee women to seek help. There is a long queue in getting a specialist appointment or even at hospitals.

"In Middle East, they don't have appointments. When you are sick, you go, and you wait over there in the reception for example until your turn is coming. So, they don't like a long waiting." (SP 3)

"My son, he needed an operation for his hip, now we are waiting for 2 years. And there is no appointment for me." (RW 2)

\subsubsection{Social and Cultural Aspects}

(a) Different Understandings of Mental Health

When asked about what mental health and wellbeing means to them, most of the refugee women could not express their knowledge very well. Sensing a lack of overall well-being or being stressed were not viewed as mental health problems. Several refugee women who were interviewed did not use any 
word consistently to express mental health issues. For example, they did not know what stress or depression means.

"I don't understand what the mental healthcare issues or mental health issues. I have not fully understood what mental health is. And where is the services." (RWI)

Mental illness is seen as a western concept by many refugees. It is a common practice in their culture to seek help from family, friends, or spiritual leaders first instead of seeing a medical professional. The refugee women mentioned that back home people with mental illness are being labeled as crazy, kept in isolation. At times the illness is described as 'nothing can be done as God has created people like this'. Therefore, many are not even willing to express any kind of mental health issue. The service providers had recognized the differences in understanding and expressing mental illness between Western and Middle Eastern culture.

"I guess, it also has to do with the culture. In western culture, we are more focused on our own mental health. Probably for their culture, if your family is doing well that means you are doing well. They might have an underlying mental health issue, but their mental health would be more focused on their family's mental health. And, I think that's cultural." (SP 5)

"I like to say something, in the back home when people see someone suffer from the mental health issue, they know this is something that we have no control over it. And, God creates those people like that." (RW 6)

Service providers corroborated the differences in understanding mental health as being a barrier to accessing services. Notably, this barrier was also partially attributed to the limited educational background of refugees rather than solely a cultural difference.

"At the beginning when they (clients) come, they don't understand anything about that one (therapy sessions). For example, if I tell them, okay I will refer you to the therapists, they don't understand. Especially when we are dealing with people with no educational background, they understand nothing. So, I have to tell them in a very simple way that, this is something related to mental health illnesses. Then they will go." (SP 3)

\section{(b) Lack of Culturally Competent Care}

The lack of culturally competent care is a significant barrier in accessing mental healthcare services for refugee women. The refugee women have a limited understanding of the 'Western' or biomedical approach to treatments for mental illness. Similarly, many service providers may also be unable to recognize or understand the way refugee clients express their mental illness. It could be difficult for a physician to read the body language or the role of culture in diagnosing or treating mental illness when not familiar with that culture.

"So, we got to be also careful how much do we ask about their past and their trauma. We've got to be sensitive to that. I mean not that such a cultural, but that may be in sense of how much we actually ask about, what the base to be able to make a diagnosis right. So how do we engage people in a respectful manner without re-traumatizing them all over again? And that's one of the challenges." (SP 2) 
The service providers have talked about the importance of teamwork. The 'One size fits all' type of care may not work for these refugee women and will further limit their access to mental healthcare.

"You can't put them all in one bag, each culture is different, each refugee is different, they have different backgrounds. I think, you just have to tailor to that individual, family or need." (SP 5)

Not being heard or having a feeling of neglect has resulted in trust issues among the refugee women about the healthcare services in general. When asked during the interview about seeking help, several refugee women said they do not think that there is anyone who will listen to them, therefore, they do not feel like sharing their problems.

"If I come here (the settlement organization) they understand me because of the language or the attitude. But for the doctor, he never asks me, I never told him anything. Just that what I complained about. He never asked me about my feeling or how do I feel." (RW 1)

"I need something to get rid of my stress. If I go to a doctor or psychologist will they guide me somehow? Do you think they will help me? I never think there are people to listen." (RW 3$)$

Preference for a female physician by refugee women is very common, which is related to their religious beliefs and Middle Eastern culture. During the interviews, it was mentioned by both refugee women and service providers that seeking permission from their husband was required to see a male physician. In some instances, the unavailability of a female physician resulted in restrictions or cancellations of appointments.

"...to make a referral for a woman to see a male doctor, with a male psychiatrist/ psychologist this woman, in some instances, has to have permission from their husband. Or they may even decline the appointment because they are scared what the community may say about." (SP 1)

Moreover, the healthcare service providing organizations including clinics and hospitals have many female service providers working there anyway. Therefore, some systematic adjustments in accommodating requests can easily be made in some circumstances and help to a great extent. However, many participants reported that this gender dynamic is changing after spending some time in Canada and the refugee women are more willing to see male physicians without any restrictions from family members. They had recognized the cultural differences with respect to gender in Canada.

\section{(c) Differences in Expectations}

The refugee women had talked about their frustrations while receiving services from healthcare providers. In some instances, women perceived that their Family Physician did not document sufficient history or engaged in conversation, which contributed to them feeling neglected. Again, the need for culturally competent care may be related here. Physicians may need to spend more time with refugee patients to build rapport and allow sufficient time for patients to express their issues. In some instances, the refugee women felt that they could not open up to the physician, therefore, a referral to a specialist was not made. During the interview one refugee woman discussed her dissatisfaction with a visit to a clinic; she was given a physical checkup but was not asked any questions about her mental wellbeing or stresses of her life. 
"If we go to physio/ family physician and there is anyone to check, they do check up the body. Why they don't check up on our mental health, if we have some mental stress or situation, talk with us to know what we are suffering from?" (RW 2)

"Last time when I was very sick, my blood pressure went up, we went to the doctor. The doctor asked why your blood pressure is high? Then I give him little details about my situation, he was not continue to ask me or refer me to anyone. That's it. He just changed the medication. Because he didn't continue or ask about my situation, that affected me negatively, so I just kept quiet." (RW 3)

(d) Stigma

Stigma is a major barrier to access that was mentioned repeatedly in the interviews. In many instances, although the refugee women had suffered significant mental health issues, they decided to manage their life stresses by themselves, and keep going or deal with it. Much of this stigma came from the negative attitude that the refugee women had faced or observed around mental illness in their country and culture. Although these Syrian refugee women have moved to Canada, the stigma about mental illness that was present at home persisted, therefore many are suffering in silence and unwilling to seek any help. Even among those who visit a Psychiatrist, many are not willing to take any medication.

"Stigma plays a significant role, I think, in individuals. Either one is not wanting to be on medication or what that means seeing a psychiatrist could be very stigmatizing. " (SP 2)

"I have clients, they have mental health issues, but they don't want me to refer them to another organization. Especially when they hear about a mental health issue, they don't want to go there. Because they are afraid how society will look at them." (SP 3)

The service providers have identified that it is very difficult for a refugee woman to talk about histories of sexual assault, as a high level of trust is required. Sexual violence carries its own stigma, in addition to mental illness.

(e) Discrimination

Negative attitudes and behavior towards refugees are common in host countries. Such behavior can create isolation, inequality of care, and further limit the refugee women's access to mental healthcare services. Racism and discrimination faced by immigrants and refugees at doctor's offices have already been reported in Canadian studies. This negative attitude or behavior towards refugees can further exacerbate their stigma, as found during the interviews. The service providers talked about discrimination among employers in recruiting refugees.

"And then, not everybody looks out at people who are different as that they belong here. There is a section of the society who feels like why they (refugees) are here. So, they (refugees) face that kind of discrimination as well. And I know one of our client's experience. Somebody on the street was very bad to her and shouted slurs to her and all of that. So that happens a lot, especially because many refugee women wear the hijab (headscarf), they are identifiable, so they do face discrimination." (SP 4)

"But at the same time, there is a lot of work that needs to be done within the mainstream community. You know it's sessions for employers because employers discriminate in hiring them as well, right. Sessions with employers, where we can talk about working with Muslims." (SP 3) 
(f) New Life and Unfamiliar Environments

Adjusting to new life, an unfamiliar environment, and the cold weather in Canada turned out to be a significant challenge for the refugee women. The lifestyle in Canada is much different than back home in Syria. Back home most of the refugee women were dependent on their husbands, they did not need to work, and focused on the care of their family. They had the traditional support of the extended family. Here in Canada, many felt the need to work outside the home, as well as be primarily responsible for taking care of the family, without the support of extended family. The situation for single, or widowed, refugee women is even more challenging as they have to do everything by themself (household chores, school appointments, doctor's appointments, etc.) in addition to their full/part-time jobs or attending EAL classes. Burdened with so many responsibilities, many are dropping out of EAL classes, cannot focus on studies, and therefore, are unable to improve their communication skills, which could have improved access to healthcare or better employment.

"I have a stressful life here. Before I was depending on my husband. Back home I didn't need to work. I only need to take care of the family. But here I should work and take care of the family. So, I have stress. My life is very stressful. And my lifestyle is changed." (RW 3)

"We try our best to learn the language, we go to school. But we found that we can not concentrate, how can I concentrate? Because the teacher needs homework from the children. For each child, I have to take care of them, I have to be with them. I have my teacher (EAL classes), I have my own homework, I have house chores, I have other works and a lot of things. How can I concentrate and focus on which one?" (RW 6)

The changed weather conditions, especially the cold winter months, came as a shock to many. Winnipeg is one of the coldest cities in Canada, with average daily high/low temperatures being $13.2^{\circ} \mathrm{C} /-23.6^{\circ} \mathrm{C}$ in January and $-9.7^{\circ} \mathrm{C} /-20.6^{\circ} \mathrm{C}$ in February [30]. It is very difficult for the refugee women, who are already depressed, to adjust to the weather conditions since their outdoor activities are even more limited during this time. Most of the refugee women do not drive and waiting for buses in the cold for appointments is not always ideal.

"I was shocked with the weather, cold weather. I never saw this winter or snow before. That made me frustrated and think about home and crying. I had the idea of cold, but not this cold." (RW3)

\section{Discussion}

In this study, we examined Syrian refugee women's experiences in accessing and utilizing mental healthcare services in Winnipeg. Most cited barriers in accessing mental healthcare services by refugee women during the interviews were language, weather, employment, and income level, stigma, and system navigation. The service providers mentioned stigma and lack of resources to provide culturally competent care. The study found similarities in the barriers that refugee women face in Winnipeg when compared to the literature in Canada and elsewhere [5]- [8], [10], [13], [31]. However, specifically for Winnipeg, the cold winter exacerbates the challenges. During the interviews, the refugee women repeatedly mentioned weather challenges, healthcare system unfamiliarity, complexity, and navigation issues. Moreover, most services are located in or near downtown Winnipeg, not in other parts of the 
city. Therefore, for the refugee women who do not drive, which is most of them, using public transport can take a long time to access a service location.

Not speaking the English language was cited as the primary barrier in accessing services by almost all interview participants. This finding is supported by similar studies conducted in Canada [7], [10], [14], [20]. The refugee women mentioned their challenges of participating in language classes while taking care of their children or if their husbands are at work. This is even more difficult for single refugee women with children and with children with disabilities in some instances. The reduced number of EAL class offerings (only one time instead of two on select days) further limited accessibility by the refugee women. Participants requested more flexibility in terms of time and locations, and facilities for childcare while attending classes, etc. Since transportation is a major issue for refugee women, offering multiple programs at one facility could be an excellent option. For example, if the refugee women can shop 'halal' food or get any skills training while they attend language classes, this can reduce their number of trips to a great extent.

Lack of cultural diversity among service providers was mentioned by participants. Moreover, the Syrian refugee women are not comfortable discussing their past experiences of physical or sexual abuse in front of a male figure, whether a physician, counsellor, or interpreter. There is stigma, and fear of shame. Service providers who are familiar with the cultural backgrounds of Syrian refugee women may be able to provide better assistance in overcoming this barrier. There is a need for female healthcare professionals and interpreters. Researchers suggested that healthcare providers need to keep in mind the circumstances around pre-and post-migration [32]-[35].

Studies have reported discrimination faced by refugees, even at doctor's office based on race, ethnicity, language, accent, religion, culture, and other characteristics [36], [37]. According to Pollock et al. (2012), the documented discrimination in accessing healthcare services includes incidents of insensitive, unfriendly, or ignorant treatment from providers to racial slurs, stereotyping, and receipt of inferior care [37]. In many cases, the religious or cultural beliefs and needs of refugee women, such as the preference for female providers or for privacy and remaining clothed were not adequately addressed [38]. The Syrian refugee women faced racism, discrimination, and bullying especially due to the fact that they wear 'hijab', and thus are identifiable. Such a negative attitude adds additional mental stress to women who are already traumatized. Every individual in Canadian society has a responsibility to address this. Public education with the use of media may be effective in eliminating such Islamophobic beliefs and behaviors.

Experiencing war-related trauma and the hardships of losing one's home have profound, diverse, and long-lasting effects on mental health beyond the acute traumatizing phase. While refugees need support with PTSD, depression, anxiety disorders, and prolonged mental illness, stigma is a major factor in preventing refugees from seeking help and accessing services. The recently published Chief Public Health Officer's Report on the State of Public Health in Canada had a focus on stigma and discrimination towards persons with health conditions [39]. The report admits that "many forms of stigma that intersect in complex ways, are very much present in our health system, driving those most in need from getting effective care and accessing services". Unfortunately, there are not many examples of successful approaches to healthcare service delivery that have eliminated stigma, especially when this culturally and linguistically diverse group is considered [35]. Salami et al. (2019) recommend using culturally appropriate terminology to refer to mental health, honoring confidentiality, and building trust with clients to help with overcoming the stigma among refugees and immigrants [31]. Furthermore, the study 
authors recommend that meeting other needs (related to social determinants of health) will set the groundwork for addressing more taboo topics like mental health.

The refugee women are also unable to express their concerns due to language and cultural barriers. The use of interpreter services or cultural brokers may mitigate these barriers but would not completely eliminate these challenges as information is still lost in translation. Moreover, there are concerns around confidentiality, misinterpretation, and availability. Additional research is needed to develop and evaluate programs and interventions to address mental health stigma, particularly among refugees. Studies have suggested training for personnel who work as service providers, interpreters, or cultural brokers [13], [31]. Such training would enable them to identify and discuss the unique mental health stressors experienced by their refugee clients as well as guide them through system navigation for mental health and social services supports. A secondary research objective of this larger study included examining refugee women's and service provider's perspectives on improving mental healthcare for refugee women in Winnipeg, Manitoba [40]. In brief, we recommended that service providers use resources developed by UNHCR and Canadian physicians in providing culturally competent care, decision-makers take leadership roles in implementing better collaboration among agencies, employers be open in hiring refugees and everyone in the society ensures that the refugee women feel welcomed and included.

\section{Conclusion}

Given the increasing number of refugees settling in Manitoba and the potentially challenging mental health issues they may face, it is important that the provincial healthcare system is well-equipped to address this potential challenge. Results from this study will assist the decision-makers in developing policies to improve mental healthcare for refugees in Manitoba. While designing programs for refugee women or providing services to them, it is important to keep in mind their pre-migration literacy level. We recommend that decision-makers and program planners consult with refugee women in program design. It is important to hear directly from those who are receiving the services, not only those who are providing the services. Many Syrian refugee women have lived through war violence, but they showed strength and they survived. This reminds us of women's resilience and spirit, and to celebrate their strength. Manitoba can take pride as a host of these brave women, as well as learn from their spirit of survival.

\section{Ethical statements}

Ethics approvals were obtained from (1) Health Research Ethics Board (HREB), University of Manitoba and (2) Research Access and Approval Committee, Winnipeg Regional Health Authority (WRHA). (Approval date: November 15, 2018; Approval Number: H2018:406).

The compliance to the Research and Publication Ethics: This study was carried out in accordance with the rules of research and publication ethics.

\section{Acknowledgments}

The authors acknowledge financial supports provided by the Canadian Institute of Health Research (CIHR) Canada Graduate Scholarship, Manitoba Training Program (MTP) for Health Services 
Research Fellowship, and the University of Manitoba Faculty of Graduate Studies top-up funding to Sanjida Newaz. Natalie Riediger is the recipient of a CIHR Early Career Investigator Award.

\section{References}

[1] UNHCR. Figures at a Glance. United Nations High Commissioner for Refugees. https://www.unhcr.org/figures-at-a-glance.html. Published in 2019. (accessed March 25, 2020)

[2] IRCC. Welcoming 25,000 Syrian refugees in 100 days. Immigration, Refugees and Citizenship Canada.https://www.canada.ca/en/immigration-refugees-citizenship/services/refugees/welcomesyrian-refugees/how.html. Published 2019. (accessed April 17, 2020).

[3] IRCC. WelcomeRefugees : Key figures Welcomed in Canada. Immigration, Refugees and Citizenship Canada. https://www.canada.ca/en/immigration-refugeescitizenship/services/refugees/welcome-syrian-refugees/key-figures.html. Published 2017. (accessed April 11, 2020).

[4] Hassan G, Kirmayer L, Mekki-Berrada A, et al. Culture, Context and the Mental Health and Psychosocial Wellbeing of Syrians. Geneva; 2015.

[5] Pavli A, Maltezou H. "Health problems of newly arrived migrants and refugees in Europe", $J$ Travel Med., 24(4), 1-8, 2017. doi:10.1093/jtm/tax016

[6] Awuah-mensah L. Examining the Experiences of Government Assisted Refugee Women with Settlement Services in Kitchener-Waterloo (Master's thesis). 2016.

[7] Sherzoi O. Photovoice: Exploring Immigrants and Refugees' Perceptions and Access to Mental Health Services in Winnipeg (Master's thesis). 2017.

[8] McKeary M, Newbold B. "Barriers to care: The challenges for Canadian refugees and their health care providers", J Refug Stud., 23(4), 523-545. 2010. doi:10.1093/jrs/feq038

[9] Sullivan C. Responding to the Complex and Gendered Needs of Refugee Women. Affil J Women Soc Work.;24(3), 272-284, 2009.

[10] Edge S, Newbold B. "Discrimination and the health of immigrants and refugees: Exploring Canada's evidence base and directions for future research in newcomer receiving countries", $J$ Immigr Minor Heal. 15(1), 141-148, 2013 ;doi:10.1007/s10903-012-9640-4

[11] Delara M. "Social determinants of women's mental health", J Psychosom Res., 63, 223-224, 2010. doi:10.1016/j.jpsychores.2007.07.002

[12] Donnelly TT, Hwang JJ, Este D, Ewashen C, Adair C, Clinton M. "If I Was Going to Kill Myself, I Wouldn't Be Calling You. I am Asking for Help: Challenges Influencing Immigrant and Refugee Women's Mental Health", Issues Ment Health Nurs., 32(5), 279-290, 2011. doi:10.3109/01612840.2010.550383

[13] Thomson MS, Chaze F, George U, Guruge S. "Improving Immigrant Populations' Access to Mental Health Services in Canada: A Review of Barriers and Recommendations", J Immigr Minor Heal., 17(6), 1895-1905, 2015. doi:10.1007/s10903-015-0175-3 
[14] Cho J. Exploring the Health and Health Care Experiences of Refugee and Refugee Claimant Women in Hamilton, Ontario: A Qualitative Study. 2012.

[15] IRCC. Annual report to parliament on Immigration, 2012-2017. Immigration, Refugees and Citizenship Canada. https://www.canada.ca/en/immigration-refugeescitizenship/corporate/publications-manuals.html. Published 2017. (accessed March 28, 2020).

[16] Government of Manitoba. Province announces new provincial health organization with a focus on patient-centered planning [Press release].http://news.gov.mb.ca/news/print,index.html?item=41692. Published June 28, 2017.

[17] Government of Manitoba. Province awards contract to develop mental health and addiction strategy [Press release].http://news.gov.mb.ca/news/index.html?item=41471. Published May 3, 2017.

[18] Kavanagh S, Marcoux J. Province plans to cut civil service by $8 \%$ following release of KPMG fiscal review. $C B C$. http://www.cbc.ca/news/canada/manitoba/kpmg-financial-review-released1.4320129. Published October 3, 2017.

[19] Asaam HM. Mental Health and Well-being of Refugees to Canada: A Brighter Future (Master's Thesis). 2015.

[20] Woodgate RL, Busolo DS, Crockett M, Dean RA, Amaladas MR, Plourde PJ. "A qualitative study on African immigrant and refugee families' experiences of accessing primary health care services in Manitoba, Canada: it’s not easy!", Int J Equity Health.,16(1), 5, 2017. doi:10.1186/s12939-016-0510-x

[21] WRHA. Optimizing The Mental Health and Emotional Wellbeing of Immigrants and Refugees in Winnipeg : A Conceptual Framework. Winnipeg; 2014.

[22] Manitoba Labour and Immigration. Manitoba Immigration Facts - 2014 Statistical Report.; 2015. http://www.immigratemanitoba.com/wp-content/uploads/2015/09/MIF2014_E_Web_Programmed.pdf.

[23] 211 Manitoba. BridgeCare Clinic. http://mb.211.ca/program-at-site/bridgecare-clinic-atdowntown-community-office-elgin-ave-site/. Published 2018. (accessed March 19, 2020).

[24] Personal-Communication. Discussions with the staff. Bridge Care Primary Care Clinic. 2019.

[25] Vasilevska B. Refugee Mental Health Practices in Canada: A Review of the Literature.; 2010.

[26] Silove D, Ventevogel P, Rees S. "The contemporary refugee crisis: an overview of mental health challenges", World Psychiatry, 16(2), 130-139, 2017. doi:10.1002/wps.20438

[27] Braun V, Clarke V." Using thematic analysis in psychology Using thematic analysis in psychology", Qual Res Psychol., 3(2), 77-101, 2006. doi:10.1191/1478088706qp063oa

[28] Vaismoradi M, Turunen H, Bondas T. "Content analysis and thematic analysis: Implications for conducting a qualitative descriptive study", Nurs Heal Sci., 15(3), 398-405, 2013.

doi:10.1111/nhs. 12048 
[29] Lincoln YS, Guba EG. Naturalistic Inquiry. Newbury Park, CA: Sage Publicationc, Inc., California, USA; 1985.

[30] weather.com. Winnipeg, Manitoba, Canada Monthly Weather.

[31] Salami B, Salma J, Hegadoren K. "Access and utilization of mental health services for immigrants and refugees: Perspectives of immigrant service providers", Int J Ment Health Nurs., 28(1), 152-161, 2019. ; doi:10.1111/inm.12512

[32] Zunzunegui MV, Forster M, Gauvin L, Raynault MF, Douglas Willms J. "Community unemployment and immigrants' health in Montreal", Soc Sci Med., 63(2), 485-500, 2006. doi:10.1016/j.socscimed.2006.01.004

[33] O'Mahony JM, Donnelly TT. "The influence of culture on immigrant women's mental health care experiences from the perspectives of health care providers", Issues Ment Health Nurs., 28(5), 453471, 2007. ;doi:10.1080/01612840701344464

[34] Rousseau C, Drapeau A. "Premigration exposure to political violence among independent immigrants and its association with emotional distress", J Nerv Ment Dis.,192(12), 852-856, 2004. doi:10.1097/01.nmd.0000146740.66351.23

[35] Guruge S, Wang AZY, Jayasuriya-Illesinghe V, Sidani S. "Knowing so much, yet knowing so little: a scoping review of interventions that address the stigma of mental illness in the Canadian context", Psychol Heal Med., 22(5), 507-523, 2017. doi:10.1080/13548506.2016.1191655

[36] Pollock G, Newbold KB, Lafreniere G, Edge S. Perceptions of discrimination in health services experienced by immigrant minorities in Ontario. 2011.

https://webaccess.wlu.ca/documents/48207/discrimination_\%26_health-final.pdf.

[37] Pollock G, Newbold K, Lafrenière G, Edge S. "Discrimination in the Doctor's Office: Immigrants and Refugee Experiences", Crit Soc Work., 13(2), 60-79, 2012. http://www.uwindsor.ca/criticalsocialwork/discriminationindoctoroffice.

[38] Reitmanova S, Gustafson DL. "They can't understand it": Maternity health and care needs of immigrant Muslim women in St. John's, Newfoundland", Matern Child Health J., 12(1), 101-111, 2008. doi:10.1007/s10995-007-0213-4

[39] Tam T. Addressing Stigma: Towards a More Inclusive Health System. The Chief Public Health Officer's Report on the State of Public Health in Canada 2019.; 2019. https://www.canada.ca/en/public-health/corporate/publications/chief-public-health-officer-reportsstate-public-health-canada/addressing-stigma-toward-more-inclusive-health-system.html.

[40] Newaz S, Riediger N. "User and Provider Perspectives on Improving Mental Healthcare for Syrian Refugee Women in Winnipeg, Manitoba, Canada", J Refug Glob Heal (in Press.;3(1), 2020). 